\title{
Changes in lower extremity function and pitching performance with increasing numbers of pitches in baseball pitchers
}

\author{
Osamu Yanagisawa*, Hidenori Taniguchi \\ Faculty of Business Information Sciences, Jobu University, Isesaki, Japan
}

Compared to upper extremity function, the changes in lower extremity function with increasing numbers of pitches have not fully been investigated in baseball pitchers. In addition, little is known about the correlation between lower extremity function and pitching performance. The purpose of this study was to evaluate the effect of fatigue on lower extremity function and pitching performance in a simulated baseball game. Eighteen collegiate baseball pitchers threw 117 pitches in 9 innings ( 13 pitches per inning) with 5 -min rest between innings at an official pitching distance in a simulated game. Isometric hip muscle strength (abduction and adduction) and squat jump performance (height, mean/peak power, and mean/peak velocity) were measured before and after the game. The mean ball velocity and pitching accuracy were assessed per inning. Ball velocity significantly decreased in the 7th $(P=0.026)$ and 9 th $(P=0.001)$ innings compared to the 1 st inning, but pitching accuracy did not change significantly. Hip abduction $(P=0.009)$ and adduction $(P=0.001)$ strength significantly decreased after the game, but squat jump performance did not significantly change before and after the game. A significant correlation between decreased ball velocity in the 9th inning and decreased hip adduction strength was found $(P=0.011, r=0.583)$. Our findings suggest that hip abduction and adduction strength are susceptible to fatigue owing to repetitive throwing motions and that hip adduction strength, especially, is an important physical fitness factor for maintaining ball velocity during a game in baseball pitchers.

Keywords: Hip muscle strength, Squat jump, Ball velocity, Pitching accuracy, Fatigue, Pitching

\section{INTRODUCTION}

Ball velocity and pitching accuracy are determining factors that strongly influence pitching performance for baseball pitchers. Several studies demonstrated a close correlation between ball velocity and ground-reaction force generated during pitching (Guido and Werner, 2012; MacWilliams et al., 1998). Therefore, greater force and power generated from the lower extremities is thought to be significant for increasing ball velocity in baseball pitching (Guido and Werner, 2012; Kageyama et al., 2014; Kageyama et al., 2015; MacWilliams et al., 1998). In addition, a stable base of the lower extremities should contribute to enhancement of pitching accuracy by supporting the powerful motions of the trunk and

upper extremities (Fortenbaugh et al., 2009; Kageyama et al., 2014; Kageyama et al., 2015; Marsh et al., 2004; Milewski et al., 2012). Moreover, it is important for baseball pitchers to keep lower extremity function at a high level throughout the course of a baseball game for better pitching performance.

Several previous studies (Escamilla et al., 2007; Grantham et al., 2014; Murray et al., 2001) have investigated changes in biomechanical parameters of the lower extremities during pitching and ball velocity with increasing numbers of pitches in baseball pitchers. Murray et al. (2001) showed significant decreases in knee angle at ball release and ball velocity at the last inning of a simulated game in professional baseball pitchers. These findings might have resulted from muscle fatigue of the lower extremities due to

${ }^{*}$ Corresponding author: Osamu Yanagisawa (D) https://orcid.org/0000-0001-8125-7833 Faculty of Business Information Sciences, Jobu University, 634-1, Toyazuka-machi, Isesaki, Gunma 372-8588, Japan

Tel: +81-270-32-1011, Fax: +81-270-32-1126, E-mail: yanagisawa@ic.jobu.ac.jp

Received: April 5, 2018 / Accepted: April 19, 2018 
repetitive pitching motions. Although Mullaney et al. (2005) found minimal muscle fatigue in the hip musculature after pitching $7 \pm 2$ inning, pitching performance was not investigated in their study. To our knowledge, very few attempts have been made to quantify the changes in lower extremity function and pitching performance with increasing numbers of pitches in baseball pitchers. It is possible that lower extremity muscle fatigue diminishes energy transfer from the lower extremity to the trunk during baseball pitching, resulting in decreased ball velocity. Moreover, a fatigued lower extremity may adversely affect pitching accuracy.

The purpose of this study was to evaluate the effect of fatigue on lower extremity function and pitching performance in a simulated baseball game and to assess the correlation between lower extremity function and pitching performance. These data would be useful for constructing training, conditioning, and rehabilitation programs for baseball pitchers. Our hypothesis was that baseball pitchers would show significantly decreased lower extremity function and pitching performance in the game and that significant correlations would be found between lower extremity function and pitching performance.

\section{MATERIALS AND METHODS}

\section{Participants}

Eighteen overhanded collegiate male baseball pitchers (righthanded, $\mathrm{n}=12$; left-handed, $\mathrm{n}=6$; mean and standard deviation [SD]: age, $19.9 \pm 0.7$ years; height, $177.8 \pm 4.8 \mathrm{~cm}$; body mass, $75.9 \pm 4.9 \mathrm{~kg}$ ) participated in this study immediately after the end of their spring baseball league games. All participants had no pain or discomfort in their body at the time of measurement and no previous history of extremity surgery or injury.

This study was approved by the institutional ethics committee of Jobu University (approval number: No.16-B01). Before the measurement, the purpose, potential risks, and examination procedures of this study were explained to all participants. Written informed consent was obtained from each participant, and the participants' rights were fully protected.

\section{Pitching protocol}

We attempted to establish a pitching protocol similar to a single game situation as much as possible. After an adequate warmup, each participant was asked to throw a baseball from a pitching mound to a catcher (the distance between a pitcher's plate and a home plate was $18.44 \mathrm{~m}$ : official pitching distance) in all pitches. The participants were instructed to aim at the strike zone of a hand-made apparatus located in front of the home plate (Yanagisawa et al., 2018). The strike zone (length $60 \mathrm{~cm} \times$ width $43 \mathrm{~cm}$ : width of home plate) of the apparatus corresponded to that of a batter approximately $175 \mathrm{~cm}$ tall. Participants threw 13 pitches (9 fastballs and 4 curveballs) per inning from a stretch position at a rate of one pitch every $15 \mathrm{sec}$; they were instructed to throw all fastball pitches at maximal effort (as fast as possible). Pitching was completed when the participants finished 9 innings of pitching with 5-min rest between innings. The total number of pitches was 117 pitches in 9 innings.

\section{Pitching performance}

Ball velocity of a fastball was measured using a radar gun (16JYM10000, MIZUNO Corp., Osaka, Japan) from behind the catcher. Mean ball velocity was evaluated per inning. In addition, pitching accuracy per inning was assessed as the passing rate of the strike zone in 9 fastball pitches (number of strikes/9 fastball pitches $\times 100$ ).

\section{Hip abduction and adduction strength}

Isometric muscle strength of hip abduction and adduction was measured using a dynamometer (T.K.K.3367b, Takei Scientific Instruments Co., Ltd., Niigata, Japan) with the participant in a

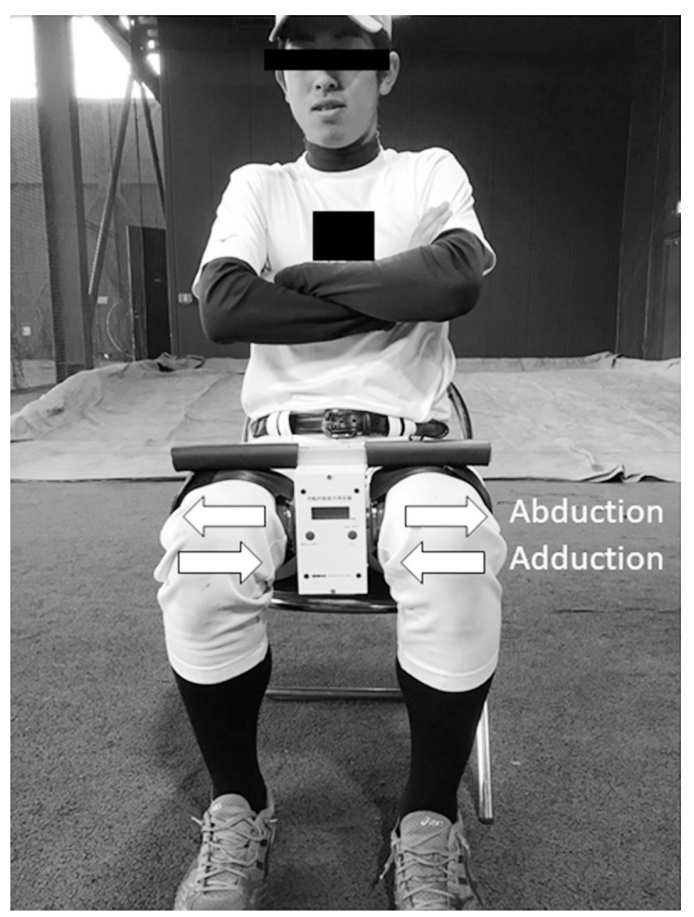

Fig. 1. Positioning for the measurement of isometric hip abduction and adduction strength. 
seated position before and after the simulated game. The dynamometer was placed between the thighs. Then, fixation belts of the dynamometer were firmly worn around the distal end of each thigh (Fig. 1). The participant was instructed to maximally perform 5-sec isometric hip adduction by pushing the pads of the dynamometer or abduction by pulling the fixation belts of the dynamometer. The calibration of the dynamometer was always performed prior to each trial. The participant performed two trials in the measurements of hip abduction or adduction strength. The maximum value of the two trials was used for analysis. The participants were always given verbal encouragement during force generation.

\section{Squat jump}

The height, mean/peak power, and mean/peak velocity for squat jump without arm swing were measured using a digital optical encoder (Gymaware, Kinetic Performance Technology, Canberra, Australia) before and after the simulated game. The optical encoder was positioned on the ground perpendicular to the movement of the body. The connection cable coupled to the encoder was attached via a tether to the belt of the participant; jump height was set to $0 \mathrm{~m}$ at upright standing position. Then, the participants performed the squat jump with their knee flexed at $90^{\circ}$, with their hands on the hips, and with the stance at hip width (Fig. 2). The examiners checked the knee angle with a goniometer before the jump and no reaction movement at the start of the jump. Par- ticipants were asked to hold the squat position for 3 sec before performing a maximal vertical jump. Additionally, they were instructed to jump as high and fast as possible. Data (jumping height, mean/peak power, and mean/peak velocity) regarding the maximal jump height of 2 trials was used for subsequent analysis.

\section{Statistical analysis}

The mean and SD were calculated for all variables. Data regarding hip strength and squat jump were compared before and after the simulated game by using a paired $t$-test. One-way analysis of variance with a Dunnett test was used to detect significant changes from 1st inning in mean ball velocity and pitching accuracy. In addition, effect sizes (Cohen $d$ ) (Cohen, 1992) were calculated and evaluated as trivial (0-0.19), small (0.20-0.49), medium (0.50$0.79)$, and large (0.80 and greater). Moreover, Pearson's correlation coefficients $(r)$ were used to evaluate the correlations between lower extremity function after the simulated game and pitching performance in the 9th inning; the rate of change in each variable was calculated. Statistical significance was set at $P<0.05$ for all analyses.

\section{RESULTS}

\section{Pitching performance}

Fig. 3 shows the changes of ball velocity from the 1st to 9th inning. Ball velocity significantly decreased in the 7 th $(128.4 \pm 5.7$
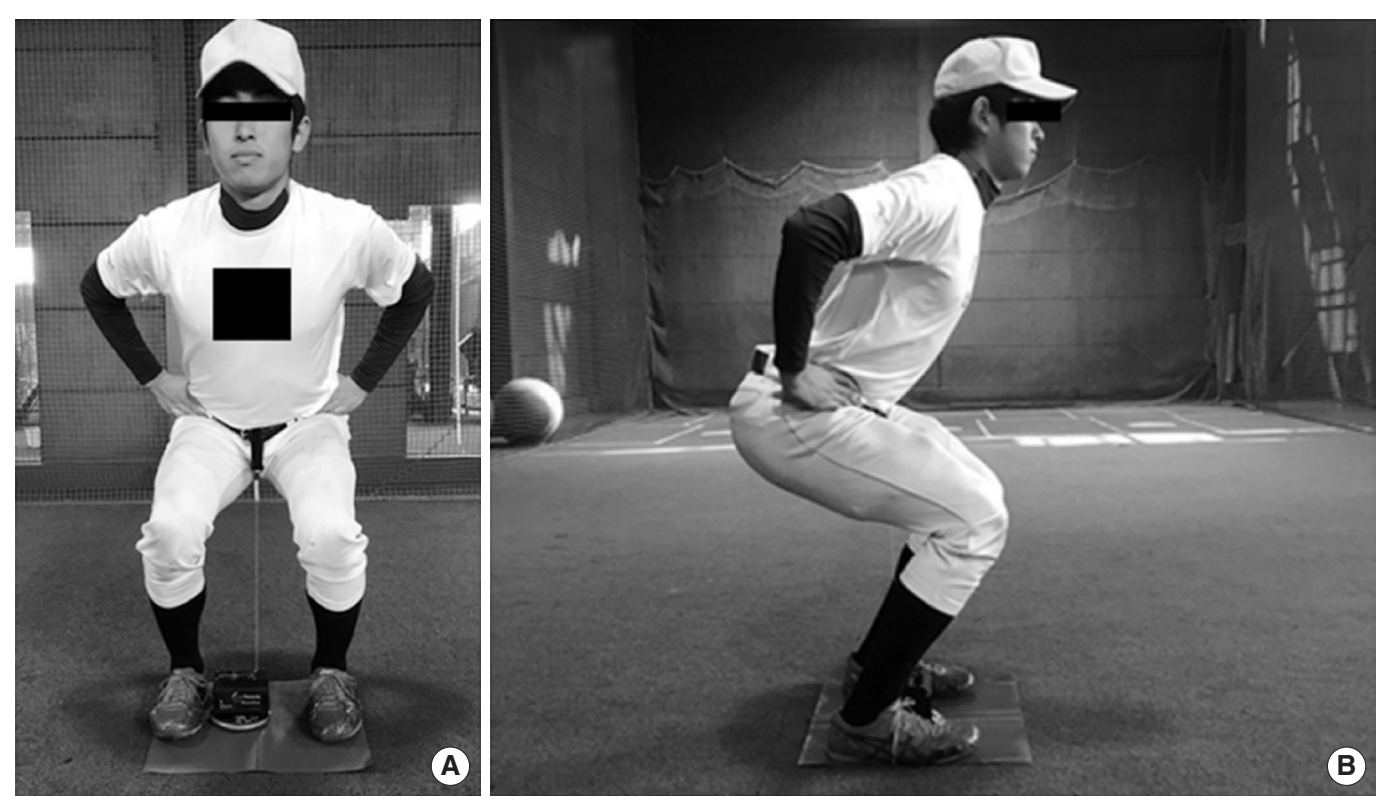

Fig. 2. Positioning for squat jump without arm swing. Frontal (A) and lateral (B) views. 


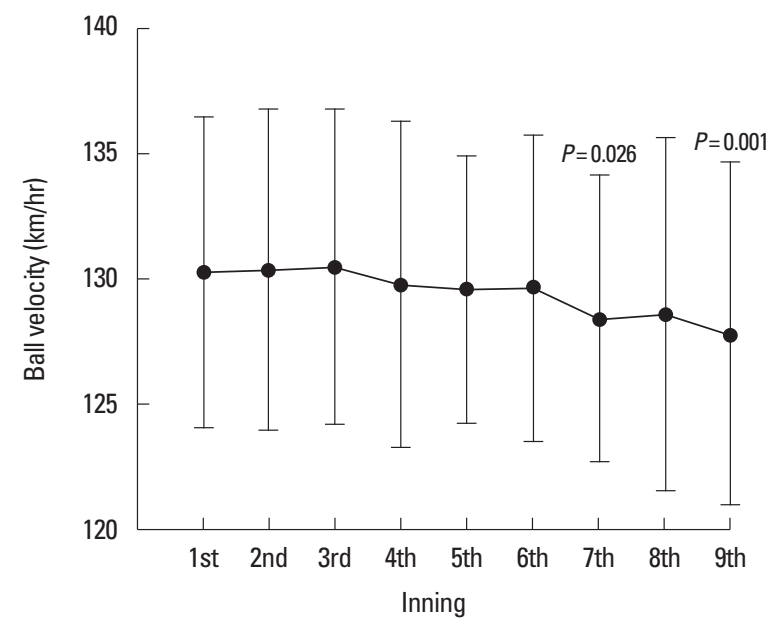

Fig. 3. Changes of ball velocity from the 1st to 9th inning (mean \pm standard deviation).

Table 1. Data regarding hip muscle strength and squat jump before and after a simulated game

\begin{tabular}{lcccc}
\hline Variable & Before & After & \multicolumn{2}{c}{ Significant Effect size } \\
\hline Hip muscle strength & & & & \\
Abduction (N) & $450.2 \pm 59.1$ & $426.7 \pm 54.9$ & $P=0.009$ & $d=0.41$ \\
Adduction (N) & $450.7 \pm 68.5$ & $421.4 \pm 54.2$ & $P=0.001$ & $d=0.47$ \\
Squat jump & & & & \\
Height (m) & $0.41 \pm 0.04$ & $0.42 \pm 0.04$ & NS & $d=0.25$ \\
Mean power (W) & $2,714.9 \pm 548.0$ & $2,704.8 \pm 572.9$ & NS & $d=0.02$ \\
Peak power (W) & $6,084.2 \pm 956.4$ & $6,291.9 \pm 1,205.6$ & NS & $d=0.19$ \\
Mean velocity (m/sec) & $1.61 \pm 0.20$ & $1.59 \pm 0.19$ & NS & $d=0.10$ \\
Peak velocity (m/sec) & $3.17 \pm 0.17$ & $3.20 \pm 0.25$ & NS & $d=0.14$ \\
\hline
\end{tabular}

Values are presented as mean \pm standard deviation.

NS, not significant.

$\mathrm{km} / \mathrm{hr}, P=0.026, d=0.32)$ and $9 \mathrm{th}(127.8 \pm 6.8 \mathrm{~km} / \mathrm{hr}, P=0.001$, $d=0.38)$ innings compared to the 1 st inning $(130.3 \pm 6.2 \mathrm{~km} / \mathrm{hr})$. On the other hand, pitching accuracy did not show significant change from the 1st inning (1st inning, $53.1 \% \pm 21.1 \%$; 2nd inning, $46.9 \% \pm 24.3 \%$; 3rd inning, $48.1 \% \pm 18.7 \%$; 4th inning, $45.1 \% \pm 16.4 \%$; 5 th inning, $48.8 \% \pm 23.8 \%$; 6th inning, $43.2 \% \pm$ $18.2 \%$; 7th inning, $45.7 \% \pm 17.8 \%$; 8 th inning, $56.2 \% \pm 21.4 \%$; 9th inning, $44.4 \% \pm 17.0 \%)$.

\section{Hip muscle strength and squat jump}

Table 1 displays the values of hip muscle strength and squat jump. Hip abduction $(P=0.009, d=0.41)$ and adduction $(P=$ $0.001, d=0.47$ ) strength significantly decreased after the simulated game. On the other hand, the height, mean/peak power, and mean/peak velocity for squat jump without arm swing showed no

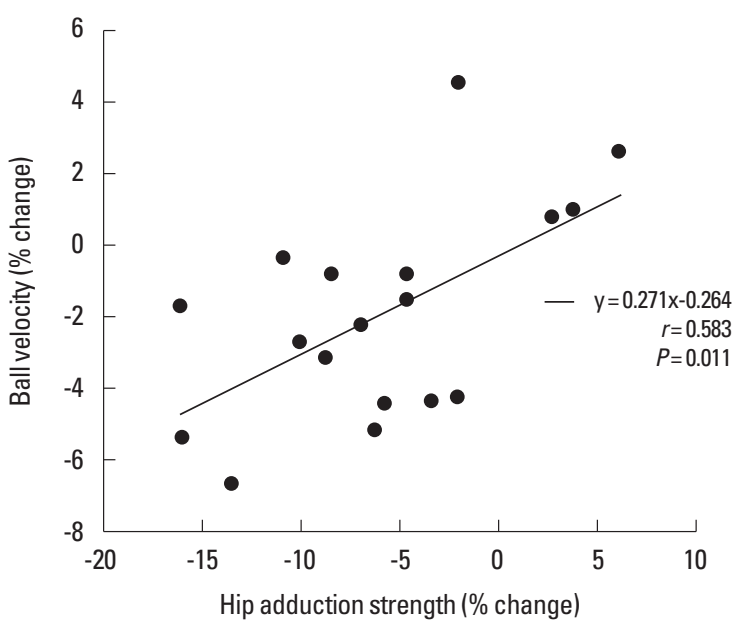

Fig. 4. Correlation of the rate of change in ball velocity in the 9th inning with hip adduction strength after the simulated game.

significant changes before and after the simulated game.

\section{Correlations between lower extremity function and pitching performance}

The simulated game resulted in significantly decreased hip abduction and adduction strength and ball velocity. A significant correlation was found between decreased ball velocity in the 9th inning and decreased hip adduction strength immediately after the game ( $P=0.011, r=0.583)$ (Fig. 4).

\section{DISCUSSION}

In the present study, hip abduction and adduction strength significantly decreased after the simulated game. These findings would be attributed to muscle fatigue resulting from repetitive throwing motions. In baseball pitching, stride length is an important biomechanical parameter for generating greater translational energy using the downward slope of the pitching mound (Dillman et al., 1993; Fortenbaugh et al., 2009; Fry et al., 2017; Milewski et al., 2012). Hip abduction strength in the pivot leg helps stabilize the pelvis and lengthen the stride by preventing downward tilt of the contralateral pelvis during the wind-up and early cocking phases of baseball pitching (Kageyama et al., 2014; Laudner et al., 2010). After stride foot contact, the stride leg generates a braking force to decelerate the motions of the lower extremities and serves as an anchor in transforming the forward and vertical momentum into rotational components (MacWilliams et al., 1998). Kageyama et al. (2014) demonstrated that hip adduction torque on the stride leg at stride foot contact was significant- 
ly greater in high-ball-velocity pitchers than in low-ball-velocity pitchers, and suggested that the greater hip adduction torque contributes to the control/stabilization of the stride leg to increase the rotation and forward motion of the trunk after the late cocking phase of baseball pitching as well as the production of greater ground-reaction force on the stride leg, resulting in the generation of high ball velocity. Similarly, the hip abductors in the stride leg also possibly contribute to the control/stabilization of the stride leg after stride foot contact (Guido and Werner, 2012; Kageyama et al., 2014; MacWilliams et al., 1998; Milewski et al., 2012). Taken together, hip abductors and adductors are thought to play important roles in the gain of appropriate stride length as well as the smooth energy transfer from the stride leg to the trunk (as a part of the entire kinetic chain) during pitching. Our findings suggest that hip adduction strength is an important physical fitness factor for baseball pitchers to maintain the ball velocity during a game. On the other hand, although Mullaney et al. (2005) found minimal muscle fatigue in the hip musculature after pitching $7 \pm 2$ inning (99 \pm 29 pitches per game), the change was not significant. Therefore, further accumulation of data is needed to clarify the effect of repetitive throwing motions on hip muscle strength in baseball pitchers.

Contrary to our expectation, in the present study all variables of the squat jump did not show significant decreases after the simulated game. Previous studies (Kageyama et al., 2014; MacWilliams et al., 1998) indicated that ball velocity correlates highly with the maximal push-off force (greater momentum by hip and knee extension) of the pivot leg generated during pitching, which supplies the initial forward momentum of the entire body in the direction of the pitch. Lehman et al. (2013) revealed that high ball velocity significantly correlated with the test score in a unilateral side jump in the frontal plane, which mimics the stride action of baseball pitching. Moreover, Murray et al. (2001) suggested that lower extremity muscle fatigue caused significant decreases in knee angle at ball release and ball velocity at the last inning of a simulated game in professional baseball pitchers. Taken together, extension power (force) of the lower extremities is thought to be a physical fitness factor that is related to ball velocity in baseball pitchers. However, the effect of repetitive throwing motions on jump performance was not found in the present study.

The present study has a few limitations. First, the lack of remarkable change in the present study may partly be attributed to limitations due to pitching in a simulated game (a noncompetitive environment) because a simulated game cannot completely replicate the conditions experimented during a competitive game
(Escamilla et al., 2007). It is possible that baseball pitchers feel more physical and/or mental fatigue in an actual game than a simulated game, resulting in more decreased muscle function and pitching performance. Second, we measured hip muscle strength and jump performance only before and after the simulated game because we thought that the number of measurements should be limited to properly assess acute physical responses (fatigue) immediately after pitching. To date, very few studies have attempted to investigate the effects of repetitive throwing motions on lower extremity function in baseball pitchers. Thus, further research is required to clarify changes in various lower extremity functions with increasing numbers of pitches in baseball pitchers at various competitive levels. In particular, physically immature baseball pitchers may be susceptible to lower extremity muscle fatigue with increasing numbers of pitches (Erickson et al., 2016). Additionally, it was revealed that lower extremity power (vertical jump height and power) decreases with age in professional baseball pitchers (Mangine et al., 2013). Third, although we did not measure upper extremity and trunk functions in this study, it is possible that muscle fatigue of the upper extremity and/or trunk muscles was related to decreased ball velocity at the end of the simulated game.

In conclusion, our data showed that hip abductors and adductors of baseball pitchers are susceptible to muscle fatigue due to repetitive throwing motions. In addition, hip adduction strength is thought to be an important physical fitness factor for maintaining ball velocity during a game in baseball pitchers.

\section{CONFLICT OF INTEREST}

No potential conflict of interest relevant to this article was reported.

\section{ACKNOWLEDGMENTS}

This study was supported by a Grant-in-Aid for Scientific Research (C) from Japan Society for the Promotion of Science. The results of the current study do not constitute endorsement of the product by the authors or the journal.

\section{REFERENCES}

Cohen J. A power primer. Psychol Bull 1992;112:155-159.

Dillman CJ, Fleisig GS, Andrews JR. Biomechanics of pitching with emphasis upon shoulder kinematics. J Orthop Sports Phys Ther 1993;18: 
402-408.

Erickson BJ, Sgori T, Chalmers PN, Vignona P, Lesniak M, Bush-Joseph CA, Verma NN, Romeo AA. The impact of fatigue on baseball pitching mechanics in adolescent male pitchers. Arthroscopy 2016;32:762771.

Escamilla RF, Barrentine SW, Fleisig GS, Zheng N, Takada Y, Kingsley D, Andrews JR. Pitching biomechanics as a pitcher approaches muscular fatigue during a simulated baseball game. Am J Sports Med 2007;35: 23-33.

Fortenbaugh D, Fleisig GS, Andrews JR. Baseball pitching biomechanics in relation to injury risk and performance. Sports Health 2009;1:314320.

Fry KE, Pipkin A, Wittman K, Hetzel S, Sherry M. Youth baseball pitching stride length: normal values and correlation with field testing. Sports Health 2017;9:205-209.

Grantham WJ, Byram IR, Meadows MC, Ahmad CS. The impact of fatigue on the kinematics of collegiate baseball pitchers. Orthop J Sports Med 2014;2:2325967114537032.

Guido JA Jr, Werner SL. Lower-extremity ground reaction forces in collegiate baseball pitchers. J Strength Cond Res 2012;26:1782-1785.

Kageyama M, Sugiyama T, Kanehisa H, Maeda A. Difference between adolescent and collegiate baseball pitchers in the kinematics and kinetics of the lower limbs and trunk during pitching motion. J Sports Sci Med 2015;14:246-255.

Kageyama M, Sugiyama T, Takai Y, Kanehisa H, Maeda A. Kinematic and kinetic profiles of trunk and lower limbs during baseball pitching in collegiate pitchers. J Sports Sci Med 2014;13:742-750.

Laudner KG, Moore SD, Sipes RC, Meister K. Functional hip characteris- tics of baseball pitchers and position players. Am J Sports Med 2010; 38:383-387.

Lehman G, Drinkwater EJ, Behm DG. Correlation of throwing velocity to the results of lower-body field tests in male college baseball players. J Strength Cond Res 2013;27:902-908

MacWilliams BA, Choi T, Perezous MK, Chao EY, McFarland EG. Characteristic ground-reaction forces in baseball pitching. Am J Sports Med 1998;26:66-71.

Mangine GT, Hoffman JR, Fragala MS, Vazquez J, Krause MC, Gillett J, Pichardo N. Effect of age on anthropometric and physical performance measures in professional baseball players. J Strength Cond Res 2013; 27:375-381.

Marsh DW, Richard LA, Williams LA, Lynch KJ. The relationship between balance and pitching error in college baseball pitchers. J Strength Cond Res 2004;18:441-446.

Milewski MD, Õunpuu S, Solomito M, Westwell M, Nissen CW. Adolescent baseball pitching technique: lower extremity biomechanical analysis. J Appl Biomech 2012;28:491-501.

Mullaney MJ, McHugh MP, Donofrio TM, Nicholas SJ. Upper and lower extremity muscle fatigue after a baseball pitching performance. Am J Sports Med 2005;33:108-113.

Murray TA, Cook TD, Werner SL, Schlegel TF, Hawkins RJ. The effects of extended play on professional baseball pitchers. Am J Sports Med 2001; 29:137-142.

Yanagisawa O, Futatsubashi G, Taniguchi H. Side-to-side difference in dynamic unilateral balance ability and pitching performance in Japanese collegiate baseball pitchers. J Phys Ther Sci 2018;30:58-62. 